\title{
Structure-to-Function Computational Prediction of a Subset of Ribosomal Proteins for the Small Ribosome Subunit
}

\author{
Edmund Ui-Hang Sim*, Chin-Ming Er \\ Department of Molecular Biology, Faculty of Resource Science and Technology, Universiti Malaysia Sarawak, \\ Kota Samarahan, Sarawak, Malaysia. \\ * Corresponding author. Tel.: +60-82-583041; email: uhsim@frst.unimas.my \\ Manuscript submitted July 18, 2014; accepted January 28, 2015. \\ doi: 10.17706/ijbbb.2015.5.2.100-110
}

\begin{abstract}
Extra-ribosomal functions of ribosomal proteins have been widely accepted albeit an incomplete understanding of these roles. Standard experimental studies have limited usefulness in defining the complete biological significance of ribosomal proteins. An alternative strategy is via in silico analysis. Here, we sought a sequence-to-structure-to-function approach to computationally predict the extra-ribosomal functions of a subset of ribosomal proteins of the small ribosome subunit, namely RPS12, RPS19, RPS20 and RPS24. Three-dimensional structure constructed from amino acid sequence was precisely matched with structural neighbours to extrapolate possible functions. Our analysis reveals new logical roles for these ribosomal proteins, of which represent important information for planning experimental and further in silico studies to elucidate their physiological roles.
\end{abstract}

Key words: Extra-ribosomal functions, RPS12, RPS19, RPS20, RPS24, structural neighbours, 3D modelling.

\section{Introduction}

Ribosomal proteins (RPs) are originally construed as only essential components of the ribosomes involved in protein biosynthesis. However, since the 1990s, their extra-ribosomal roles have been discussed revealing their association with congenital diseases and a wide range of cancers [1], [2]. For instance, over-expression of RPS12 has been observed in the tissues of colon adenocarcinomas and adenomatous polyps [3], squamous cell carcinoma of the human uterine cervix [4], and gastric cancer [5]. The expression of RPS19 was also found to be up-regulated in colon carcinoma [6], and was commonly mutated in the patients with the congenital disorder of Diamond-Blackfan Anemia [7]. Truncating germline mutations in RPS20 predisposed individuals to hereditary nonpolyposis colorectal carcinoma [8], and RPS24 showed significant differential expression between tissues of hepatocellular carcinoma and normal controls [9]. Despite these findings, knowledge on the definitive and complete functional roles of the proteins encoded by these genes remains vague. This is because their mutation status or expression behaviours in cancers alone do not provide sufficient information on their actual functions in the context of cellular development and differentiation.

Access to information on extra-ribosomal functions of these ribosomal proteins is largely hindered by the fact that existing studies focus on their expression profiles rather than biological functions. This is partly because the complete experimental characterization of RPs is laborious, time-consuming and costly. Then 
again, to our knowledge, there are very few proper studies that relate the 3D structures of RPs with their probable functions. Efforts to gain theoretical functions of proteins by considering their secondary structures are justified on the basis that the function of a protein is tightly linked to its three dimensional (3D) structure [10]. In fact, such cogitation and approach of using in silico analysis on other RPs are not unprecedented. Recently, Ref. [11] has computationally predicted interacting partners of two ribosomal proteins (RPL27 and RPL37a) and extrapolated their extra-ribosomal functions.

Herein, we will report the findings on computational derivation of the 3D models of a subset of ribosomal proteins for the small ribosomal subunit, namely RPS12, RPS19, RPS20, and RPS24. These constructed logical models, in turn, were used to predict biological functions of the proteins studied. The former effort was carried out via comparative homology modelling using the 3D-JIGSAW platform and the latter study was conducted using the strategy of structural neighbour prediction and functional matching via the Vector Alignment Search Tool (VAST) platform.

\section{Methods}

\subsection{D Structural Modelling}

The amino acid sequences of RPs were downloaded from GenBank (Accession no. CAA37582; via the National Center for Biotechnology Information (NCBI) website, http://www.ncbi.nlm.nih.gov/) in FASTA format and was submitted to a protein comparative modelling server, 3D-JIDSAW (http://www.bmm.icnet.uk/ 3djigsaw/). Three-dimensional model of RPS12 based on homologues of known structures [12] was generated by this platform. In our case, the default parameters of the program were used in the analysis. Constructed logical 3D models of the RPs were viewed using the RasMol software (Version 2.7.4.2).

\subsection{Functional Extrapolation}

Functional predictions of RPs were based on firstly, identifying its structural neighbours, and secondly, using known functions of structural neighbours to derive the functions. To search for structural neighbours, constructed 3D models of the RPs were submitted as Protein Data Bank (PDB) file to the Vector Alignment Search Tool (VAST) server (http://www.ncbi.nlm.nih.gov/Structure/VAST/vastsearch.html). The algorithm of the analysis involves a search against medium-redundancy subset of PDB, and the structural alignment of the query protein with its corresponding structure neighbours [13]. Significance of the comparison is represented by the $p$-value where 0.001 indicates that the odd of a match by pure chance is $1 / 1000$. Files of the structural comparison were downloaded and viewed by Cn3D software (http://www.ncbi.nlm.nih.gov/Structure/CN3D/cn3d.shtml). The annotated databases of PDB (http://www.rcsb.org/pdb/home/home.do), PFAM (http://pfam.sanger.ac.uk; [14]), SCOP (http://scop.mrc-lmb.cam.ac.uk/scop; [15]), and CATH (http://www.cathdb.info/index.html; [16], [17]) were employed for functional annotation of the RPs in order to computationally derive logical functions.

\section{Results and Discussion}

\subsection{RPS12}

Constructed 3D model of RPS12 reveals one domain comprising six $\alpha$-helices that surround a four-stranded antiparallel $\beta$-sheet, and three small strands of $\beta$-sheet (each containing one residue only) in the middle (Fig. 1(a)). The four structural neighbours of RPS12 are C-terminal domain of human Pelota (human PELO) homologue (CGi-17), mouse GADD45 gamma (GADD45 $\gamma$, a Growth arrest and DNA-damage-inducible 45 protein family member), the human transcription initiation factor TFIIH, and the

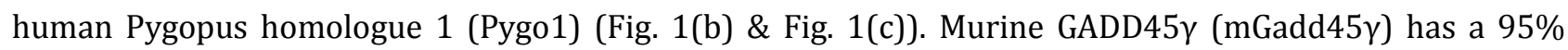


homology with its human homologue hGADD45 $\gamma$.

Both CGi-17 and GADD45 $\gamma$ have the basic structure of a four-stranded antiparallel $\beta$-sheet surrounded by six $\alpha$-helices, of which resemble the 3D model of RPS12 (Fig. 1(b)). In fact, the structural alignment among CGi-17, GADD45 $\gamma$ and RPS12 reveals similar pattern in conserved residues (red coloured residues, Fig. 1(b)), thus implying similar functions among the three proteins.

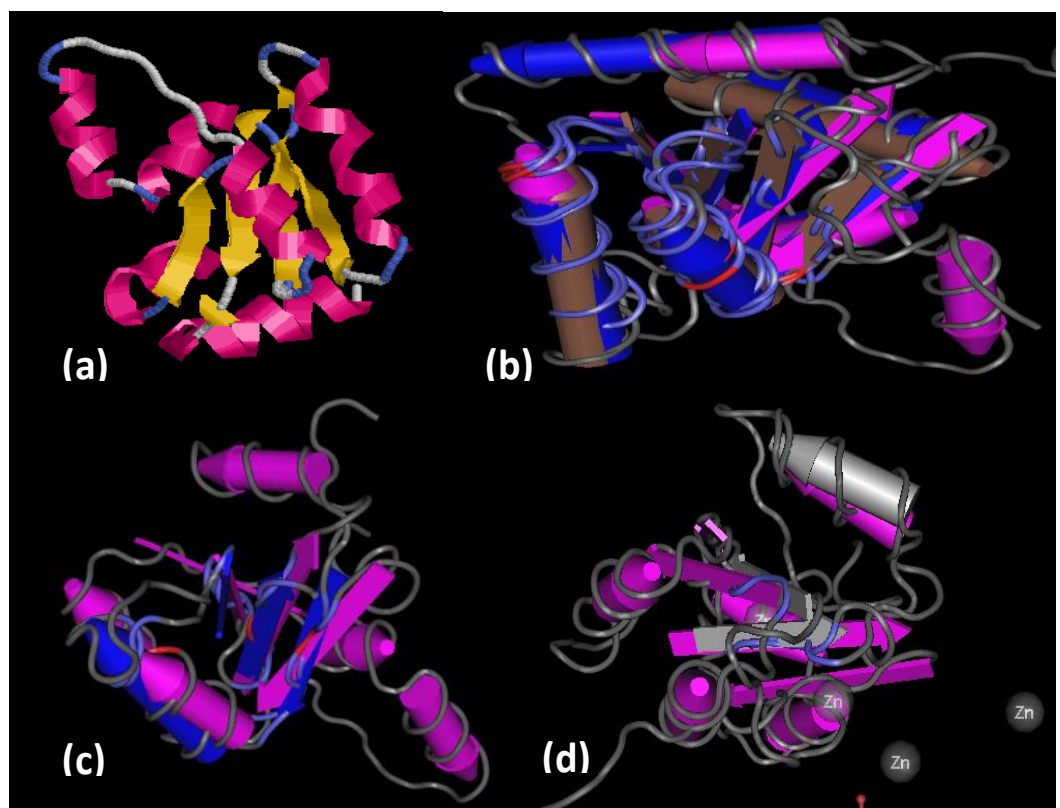

Fig. 1. Logical 3D-structure protein model of RPS12 (a); and the superposition of RPS12 (purple) with its structural neighbours of (b): murine GADD45 (blue) and C-terminal domain of human Pelota homolog (brown); (c): human general transcription factor IIH (blue); and (d): human Pygopus homolog 1 (grey).

Eukaryotic Pelota is involved in the regulation of cell cycle during cellular division [18], and mRNA translation [19]. Human Pelota (human PELO) has been shown to localize to actin cytoskeleton of mammalian cells where its over-expression and association with actin microfilament affects cell growth and spreading, and cytoskeleton organization [20]. In this respect, its interaction with some cytoskeleton-associated proteins may facilitate the detection and degradation of aberrant mRNAs [20]. On the other hand, GADD45 proteins generally mediate DNA demethylation during cell differentiation and stress response via a DNA excision repair mechanism [21]. GADD45 $\alpha$ and $\gamma$ are also required for JunD (an AP-1 transcription factor family member)-mediated induction of apoptosis in prostate cancer cells [22]. By considering the known functions of human PELO and GADD45, we infer that RPS12 could be associated with the functions of regulating cell cycle during division; modulating cytoskeleton organization; DNA demethylation via excision repair mechanism; and JunD-mediated induction of apoptosis (Table 1).

For TFIIH, of the two $\alpha$-helices and the three-stranded antiparallel $\beta$-sheets, one each of these structures aligns to RPS12 (Fig. 1C). Besides the basic role in the transcription of protein-coding genes, TFIIH has been known to be involved in DNA excision repair - the two events (transcriptional regulation and DNA repair) possibly interconnected through TFIIH's coordination [23]. The partial structural similarity between RPS12 and TFIIH suggests the former's association with transcriptional regulation of structural genes, and increases our suspicion of its role in the DNA excision repair event.

In the case of Pygo1, we also observed partial similarity in 3D pattern between its Pygopus homology domain (PHD) finger and RPS12 protein. A two-stranded antiparallel $\beta$-sheet and one $\alpha$-helix structures of Pygo1 (PHD finger) are aligned with RPS12 (Fig. 1(d)). Pygo1's involvement in Wnt-induced transcription 
has been demonstrated in the formation of a Pygo-BCL9/legless complex (binding of Pygo PHD finger to cognate HD1 domain in BCL9/legless) that preferentially binds to the histone $\mathrm{H} 3$ tail that is methylated at lysine 4 (H3K4me), in order that histone decoding can ensue [24]. Could RPS12 play similar roles as Pygo1 in facilitating Wnt-induced transcriptional regulation? Our data suggests a conceptual possibility of this.

Table 1. VAST Analysis Results on Structural Neighbours of RPS12, and Its Inferred Functions

\begin{tabular}{|c|c|c|c|}
\hline Structural neighbours & PDB ID & $p$-value & Predicted functions of RPS12 \\
\hline CGi-17 & $1 \times 52 \_A$ & 0.0011 & $\begin{array}{l}\text { Regulate translation and cell cycle; } \\
\text { modulate cytoskeleton organization }\end{array}$ \\
\hline GADD $45 \gamma$ & 3cg6_A & $10 \mathrm{e}-9.4$ & $\begin{array}{l}\text { Demethylation via excision repair; } \\
\text { facilitate JunD-mediated apoptosis }\end{array}$ \\
\hline TFIIH & $1 y d l$ & 0.0104 & $\begin{array}{l}\text { DNA excision repair; } \\
\text { transcriptional regulation }\end{array}$ \\
\hline Pygo1 & $2 \operatorname{vpg} C$ & 0.0401 & Wnt-signaling associated transcription regulation \\
\hline
\end{tabular}

\subsection{RPS19}

The constructed model of RPS19 consists of four long $\alpha$-helices, three short $\alpha$-helices (consists of 3-5 residues only) and a small two-stranded antiparallel $\beta$-sheet (Fig. 2(a)). This structure resembles a winged helix domain which consists of four helices and a two-stranded beta-sheet. The five most logical structural neighbours of RPS19 are the Z-beta Domain from the RNA-editing enzyme, Adar1 (Double-stranded RNA-specific Adenosine Deaminase); Winged Helix Domain in RNA Polymerase III 39kDa (RPC39) Polypeptide; Human ESCRT-II Complex (Vacuolar-sorting Protein, SNF8); C-terminal Domain of the human Replication Protein A 32 (RPA32) complexed with Ung2; and the Winged Helix-Turn-Helix Motif of the Human Cul-4b (Table 2). Most of these structural neighbours contain a two-stranded antiparallel $\beta$-sheet, and a minimum of three $\alpha$-helices. These secondary structures aligned neatly to the 3D model of RPS19 (Fig. 2(b)), and are classified largely as alpha protein by CATH classification. This is similar to the structure of RPS19 which consists mainly of $\alpha$-helices. The five structural neighbours provide conceptual insight into the extra-ribosomal functions of RPS19 that include transcriptional regulation, RNA processing, and DNA repair (Table 2). All of these functions are associated with DNA binding capabilities.

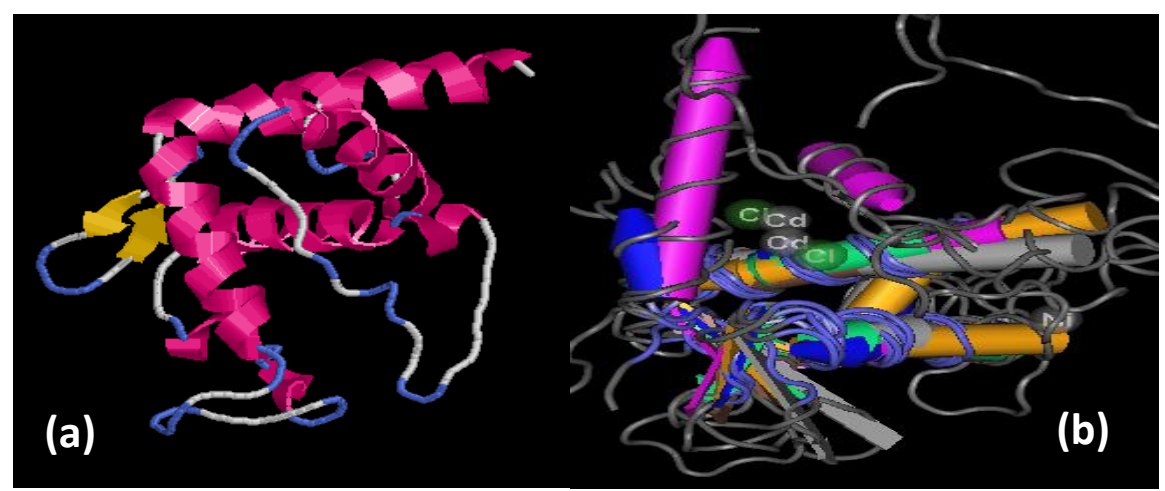

Fig. 2. (a) Constructed 3D-structural model of RPS19; and (b) the 3D superposition of RPS19 (purple) with its structural neighbours: 1xmk_A (human Adar1, blue), 2dk5_A (human RPC39, brown), 1dpu_A (human RPA32, green), 2zme_A (human SNF8, grey) and 2do7_A (human Cul-4b, orange). 
Table 2. VAST Analysis Results on Structural Neighbours of RPS19, and Its Inferred Functions

\begin{tabular}{cccc}
\hline \hline Structural neighbours & PDB ID & $p$-value & Predicted functions of RPS19 \\
\hline Adar1 & 1xmk_A & $10 \mathrm{e}-4.8$ & RNA processing \\
RPC39 & 2dk5_A & $10 \mathrm{e}-5.0$ & Transcriptional regulation \\
SNF8 & 2zme_A & 0.0004 & \\
RPA32 & 1dpu_A & $10 \mathrm{e}-5.2$ & DNA repair (in response to DNA damage) \\
Cul-4b & 2do7_A & 0.0004 & \\
\hline \hline
\end{tabular}

Since all the structural neighbours share similarity in one domain, the winged helix domain containing four $\alpha$-helices and a two-stranded $\beta$-sheet, we infer that RPS19 also consists of this domain. Winged helix domain has been known transcription factor capability because of its forkhead (Fox) box motif that can bind to DNA, and Fox proteins have been found to be important for development and disease [25]. Besides this, our data that suggest RPS19's function in RNA processing is consistent with findings of Ref. [26] which demonstrated the correlation of depleting RPS19 (and other RPs) with the increase of precursor rRNA. Hence, our in silico findings strengthen the notion of an extra-ribosomal role of RPS19 in the processing of RNA. To our knowledge, there is also no experimental evidence on association of RPS19 with DNA repair activities in human/mammals. Therefore, our results represent the first logical inference of RPS19's role in the repair of DNA.

\subsection{RPS20}

Ribosomal protein S20 is composed of a four-stranded antiparallel $\beta$-sheet, and two long $\alpha$-helices. The logical 3D model of RPS20 is relatively small and simple having an amino acid sequence of only 119 residues (Fig. 3(a)). The four most logical structural neighbours of RPS20 chosen for 3D structural alignment (with RPS20) are 1b64_A (guanine nucleotide exchange factor domain from human elongation factor - 1 beta, EF1B), 1wi8_A (RNA binding domain of the eukaryotic translation initiation factor 4B, eIF-4B), 2dnm_A (RNA binding domain in SRp46 splicing factor, SRSF), and 1j4w_A (K homology domains (KH3 and KH4) of the FUSE-binding protein, FBP) (Fig. 3(b) and Fig. 3(c)). Their known functions are translational elongation and initiation, transcriptional regulation and RNA processing (Table 3).

These structural neighbours share similar 3D pattern with RPS20, where each of them have two $\alpha$-helices and a four-stranded anti-parallel $\beta$-sheet that can align neatly with RPS20 (Fig. 3(b) and Fig. 3(c)). Classification by SCOP and PFAM indicate that these structural neighbours are alpha and beta proteins with RNA recognition motif (RRM) arranged in a ferredoxin-like fold. The presence of RRM motif confers nucleotides or polypeptides binding capabilities allowing us to infer similar behaviours for RPS20. Therefore, we predict extra-ribosomal roles of RPS20 listed in Table 3. Additionally, since the RRM motif is a conserved region in metazoan pre-mRNA splicing factor [27], the involvement of RPS20 in modulating activities of RNA processing and splicing is highly plausible.

Our data that suggest RPS20's involvement in translational initiation and elongation (Table 3, Row 1) matches its canonical roles as a component in the 40S ribosomal subunit, which is known to act as a primer for translation initiation [28]. Finally, the structural alignment of RPS20 with the Far-upstream Element (FUSE)-binding protein implies the former's association with the regulation of c-MYC expression. This is because FUSE-binding proteins have been shown to bind single-stranded FUSE region of c-MYC oncogene (Fig. 3b), presumably to regulate the expression of c-MYC [29]. The interaction between MYC protein and RPs is not an unproven phenomenon. In fact, MYC protein is known to regulate transcription of RP genes, and its expression level in tumour cells positively correlates with that of some RP genes [30]. In the case of RPS20, the up-regulation of its gene was observed in colorectal carcinoma [31], where up-regulation of 
c-MYC is also often observed [32]. Based on all these, we deductively propose RPS20 to play a role in the regulation of c-MYC transcription via binding to FUSE element of the oncogene. Recently, Ref. [33] showed that RPS20 binds Mdm2 protein to regulate the Mdm2-p53-MdmX network, thereby stabilizing endogenous p53. Here, we speculate that RPS20's extra-ribosomal roles in containing tumourigenesis are possibly via both the transcriptional control of oncogenes and the stabilization of tumour suppressors - a hypothesis that is now justified to be tested experimentally.

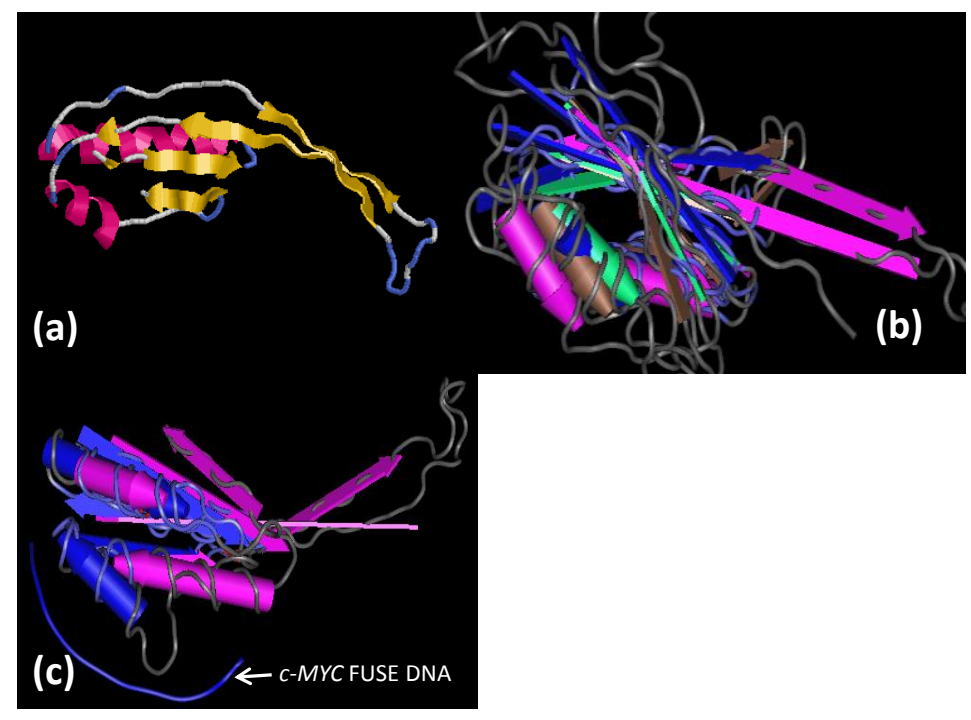

Fig. 3. (a) Constructed 3D model of RPS20; (b) - the 3D superposition of RPS20 (purple) with its structural neighbours, 1b64_A (elongation factor 1-beta, blue), 1wi8_A (Eukaryotic translation initiation factor 4B, green), 2dnm_A (SRp46 splicing factor, brown); and (c) - 3D structural alignment of RPS20 (purple) with the structural neighbour, 1j4w_A (FUSE-binding protein, blue).

Table 3. VAST Analysis Results on Structural Neighbours of RPS20, and Its Inferred Functions

\begin{tabular}{cccc}
\hline \hline Structural neighbours & PDB ID & $p$-value & Predicted functions of RPS20 \\
\hline EF1B & 1b64_A & 0.0087 & Translation elongation \\
eIF-4B & 1wi8_A & 0.0253 & Regulation of translational initiation \\
SRSF & 2dnm_A & 0.0096 & RNA splicing/mRNA processing \\
FBP & 1j4w_A & 0.0321 & Transcriptional regulation $(c-M Y C$ expression $)$ \\
\hline \hline
\end{tabular}

\subsection{RPS24}

The logical 3D structural model of RPS24 shows four $\alpha$-helices, a five-stranded antiparallel $\beta$-sheet and a small two-stranded antiparallel $\beta$-sheets (one to two residues) (Fig. 4(a)). RasMol data reveal 15 turns, as indicated by blue coloured structures in Fig. 4(a). Among the structural neighbours identified through VAST calculation for 3D model of RPS24, only two are eukaryotic factors of human origin. These were subjected to 3D superposition with RPS24, and are 2d9i_A (smr domain of NEDD4-binding protein 2) and 1msz_A (R3H domain of DNA-binding protein S $\mu \mathrm{bp}$-2) (Fig. 4(b)). The protein designated as 2d9i_A is composed of three $\alpha$-helices and a four-stranded antiparallel $\beta$-sheet, and is classified by PFAM to contain a Smr (Small MutS-related) domain. On the other hand, 1msz_A has a structure of two $\alpha$-helices and one three-stranded antiparallel $\beta$-sheet, and is classified by PFAM as an alpha and beta class of protein with a R3H domain. Both 2d9i_A and 1msz_A align with the C-terminal domain of RPS24, which is composed of two $\alpha$-helices and a two-stranded $\beta$-sheet (Fig. 4(b)). 


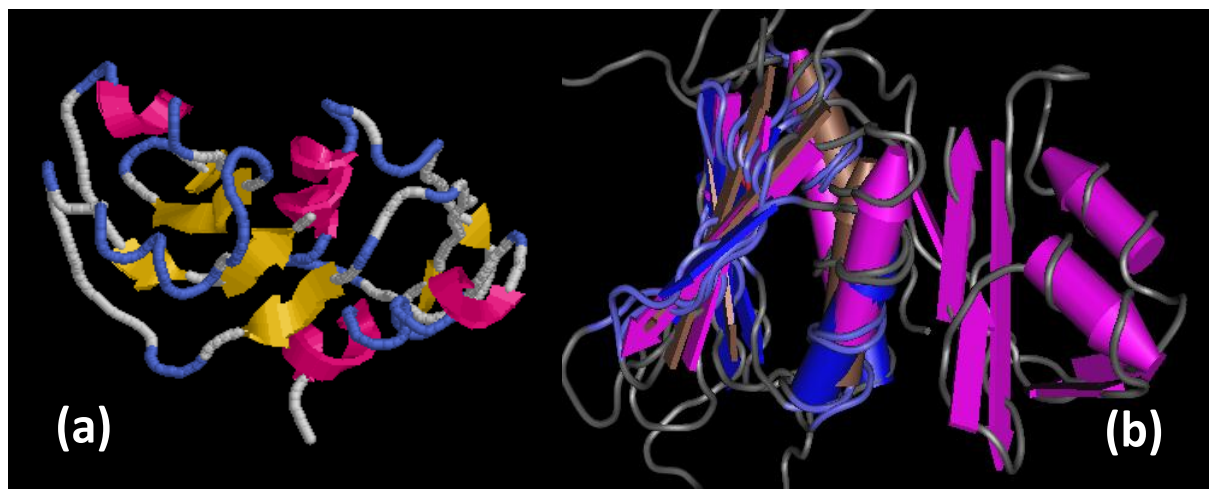

Fig. 4. (a) Constructed 3D structural model of RPS24; and (b) the 3D superposition of RPS24 (purple) with its structural neighbours: 2d9i_A (smr domain of human NEDD4-binding protein, blue) and 1msz_A R3H domain of human $\mathrm{S} \mu \mathrm{bp}-2$, brown).

NEDD4-binding protein 2, also known as NEDD4 family-interacting protein 2 (NDFIP2), binds with NEDD4 (a family of E3 ubiquitin ligases) [34] at the WW-1, -2, and -3 domains to elicit Nedd4-dependent ubiquitination pathways in human $\mathrm{T}$ lymphocytes [35]. The roles of ubiquitination in the immune system are demonstrated in apoptosis, and activation of transcription, translation, and protein kinase [36]. By parallel comparison with its structural neighbour (NDFIP2) we infer that amongst its extra-ribosomal capabilities, RPS24 could be involved in the transcriptional, translational, and protein kinase activity control within T lymphocyte physiology (Table 4).

Table 4. VAST Analysis Results on Structural Neighbours of RPS24, and Its Inferred Functions

\begin{tabular}{cccc}
\hline \hline Structural neighbours & PDB ID & $p$-value & Predicted functions of RPS20 \\
\hline NDFIP2 & 2d9i_A & 0.0094 & $\begin{array}{c}\text { Regulation of transcription, translation and } \\
\text { protein kinase (T-lymphocyte cells) }\end{array}$ \\
\cline { 3 - 4 } S $\mu \mathrm{bp}-2$ & 1msz_A & 0.0231 & $\begin{array}{c}\text { Immunoglobulin class switching } \\
\text { Transcription regulation }\end{array}$ \\
\hline \hline
\end{tabular}

Generally, R3H motifs are suspected to be associated with polynucleotide-binding [37], and human S $\mu$ bp-2 proteins were initially found to bind single-stranded DNA of the immunoglobulin $\mu$-chain switch region that has a 5'-phosphorylated guanine-rich sequence [38]. By virtue of its interaction with the genes of glial factor 1 [39], rat insulin enhancer binding protein 1 [40], cardiac transcription factor 1 [41], and also the Epstein-Barr virus lytic switch promoter [42], S $\mu \mathrm{bp}-2$ is classified as a transcription factor as well. Hence, based on the functions of S $\mu b p-2$, we infer that RPS24 would exhibit the roles of nucleic acid binding, particularly in immunoglobulin class switching and transcriptional control (Table 4).

\section{Conclusion}

The in silico analysis involving 3D protein modeling, structural neighbor prediction, and functional matching have allowed us to derive extra-ribosomal functions of RPS12, RPS19, RPS20 and RPS24. These postulated functions are summarized in Table 5. This information provides new theoretical insights into the de novo functions of this subset of ribosomal proteins. Consequently, this is important for the design of experimental studies and simulated molecular docking analysis in order to characterize the physiological roles of these proteins in the context of tumourigenesis and cellular development. 
Table 5. Summary of the Predicted Extra-Ribosomal Functions of RPS12, RPS19, RPS20 and RPS24

\begin{tabular}{lcccc}
\hline \hline Predicted functions & RPS12 & RPS19 & RPS20 & RPS24 \\
\hline Transcriptional regulation & $\checkmark$ & $\checkmark$ & $\checkmark$ & $\checkmark$ \\
Translational regulation & $\checkmark$ & & $\checkmark$ & $\checkmark$ \\
DNA repair & $\checkmark$ & $\checkmark$ & & \\
RNA processing & & $\checkmark$ & $\checkmark$ & \\
Cell cycle regulation and apoptosis & $\checkmark$ & & \\
Cytoskeleton organisational modulation & $\checkmark$ & & & \\
Demethylation & $\checkmark$ & & & $\checkmark$ \\
Protein kinase regulation & & & & $\checkmark$ \\
Ig class switching & & & & \\
\hline \hline
\end{tabular}

\section{Acknowledgment}

This study is funded by the Ministry of Education (Malaysia) under the Fundamental Research Grant Scheme [Grant no.: FRGS/ST03(01)/962/2013(03)].

\section{References}

[1] Wool, I. G. (1996). Extraribosomal functions of ribosomal proteins. Trends in Biochemical Sciences, 21, 164-165.

[2] Noara, H. (1999). Involvement of ribosomal proteins in regulating cell growth and apoptosis: Translational modulation or recruitment for extraribosomal activity? Immunology and Cell Biology, 77, 197-205.

[3] Pogue-Geile, K., Geiser, J. R., Shu, M., Miller, C., Wool, I. G., Meisler, A. I., et al. (1991). Ribosomal protein genes are overexpressed in colorectal cancer: Isolation of a cDNA clone encoding the human S3 ribosomal protein. Molecular and Cellular Biology, 11, 3842-3849.

[4] Cheng, Q., Lau, W. M., Chew, S. H., Ho, T. H., Tay, S. K., \& Hui, K. M. (2002). Identification of molecular markers for the early detection of human squamous cell carcinoma of the uterine cervix. British Journal of Cancer, 86, 274-281.

[5] Sun, X. J., Sun, K. L., Zheng, Z. H., Hao, D. M., Fu, W. N., Xu, H. M., et al. (2005). Analysis of gene expression profiles for distinct stages of intestinal-type gastric cancer using suppression subtractive hybridization and cDNA microarray. Scandinavian Journal of Gastroenterology, 40, 1244-1245.

[6] Kondoh, N., Schweinfest, C. W., Henderson, K. W., \& Papas, T. S. (1992). Differential expression of S19 ribosomal protein, laminin-binding protein, and human lymphocyte antigen class I messenger RNAs associated with colon carcinoma progression and differentiation. Cancer Research, 52, 791-796.

[7] Draptchinskaia, N., Gustavsson, P., Andersson, B., Pettersson, M., Willig, T. N., Dianzani, I., et al. (1999). The gene encoding ribosomal protein S19 is mutated in Diamond-Blackfan anaemia. Nature Genetics, 21, 169-175.

[8] Nieminen, T. T., O’Donohue, M. F., Wu, Y., Lohi, H., Scherer, S. W., Paterson, A. D., et al. (2014). Germline mutation of RPS20, encoding a ribosomal protein, causes predisposition to Hereditary Nonpolyposis Colorectal Carcinoma without DNA mismatch repair deficiency. Gastroenterology, 147(3), 595-598.

[9] Zhang, P. J., Wei, R., Wen, X. Y., Ping, L., Wang, C. B., Dong, Z. N., et al. (2012). Gene expression profiling of peripheral blood cells of patients with hepatocellular carcinoma. Cell Biology International, 36, 803-809.

[10] Peitsch, M. C. (2002). About the use of protein model. Bioinformatics, 18, 934-938.

[11] Chan, S. L. L., \& Sim, E. U. H. (2013). Bioinformatics analysis of the ribosomal proteins, RPL27, RPL37a and RPL41: 3-D protein modeling and protein-protein interaction prediction. International Journal of 
Bioscience, Biochemistry and Bioinformatics, 3, 10-15.

[12] Bates, P. A., Kelley, L. A., MacCallum, R. M., \& Sternberg, M. J. E. (2001). Enhancement of protein modelling by human intervention in applying the automatic programs 3D-JIGSAW and 3D-PSSM. Protein, Suppl., 5, 39-46.

[13] Gibrat, J. F., Madej, T., \& Bryant, S. H. (1996). Surprising similarities in structure comparison. Current Opinion in Structural Biology, 6, 377-385.

[14] Finn, R. D., Tate, J., Mistry, J., Coggill, P. C., Sammut, J. S., Hotz, H. R., et al. (2008). The Pfam protein families database. Nucleic Acids Research, 36(Database issue), 281-288.

[15] Murzin, A. G., Brenner, S. E., Hubbard, T., \& Chothia, C. (1995). SCOP: A structural classification of proteins database for the investigation of sequences and structures. Journal of Molecular Biology, 247, 536-540.

[16] Cuff, A. L., Sillitoe, I., Lewis, T., Redfern, O. C., Garratt, R., Thornton, J., et al. (2009). The CATH classification revisited - architectures reviewed and new ways to characterize structural divergence in superfamilies. Nucleic Acids Research, 37(Database issue), 310-314.

[17] Cuff, A. L., Sillitoe, I., Lewis, T., Clegg, A. B., Rentzsch, R., Furnham, N., et al. (2011). Extending CATH: Increasing coverage of the protein structure universe and linking structure with function. Nucleic Acids Research, 39(Database issue), 420-426.

[18] Eberhart, C. G., \& Wasserman, S. A. (1995). The pelota locus encodes a protein required for meiotic cell division: An analysis of G2/M arrest in Drosophila spermatogenesis. Development, 121, 3477-3486.

[19] Davis, L., \& Engebrecht, J. (1998). Yeast dom34 mutants are defective in multiple developmental pathways and exhibit decreased levels of polyribosomes. Genetics, 149, 45-56.

[20] Burnicka-Turek, O., Kata, A., Buyandelger, B., Ebermann, L., Kramann, N., Burfeind, P., et al. (2010). Pelota interacts with HAX1, EIF3G and SRPX and the resulting protein complexes are associated with the actin cytoskeleton. BMC Cell Biology, 11, 28.

[21] Niehrs, C., \& Schäfer, A. (2012). Active DNA demethylation by Gadd45 and DNA repair. Trends in Cell Biology, 22, 220-227.

[22] Zerbini, L. F., de Vasconcellos, J. F., Czibere, A., Wang, Y., Paccez, J. D., Gu, X., et al. (2011). JunD-mediated repression of GADD45 $\alpha$ and $\gamma$ regulates escape from cell death in prostate cancer. Cell Cycle, 10, 2583-2591.

[23] Compe, E., \& Egly, J. M. (2012). TFIIH: When transcription met DNA repair. Natures Review in Molecular and Cell Biology, 13, 343-354.

[24] Fiedler, M., Sánchez-Barrena, M. J., Nekrasov, M., Mieszczanek, J., Rybin, V., Müller, J., et al. (2008). Decoding of methylated histone H3 tail by the Pygo-BCL9 Wnt signaling complex. Molecular Cell, 30, 507-518.

[25] Kaestner, K. H., Knochel, W., \& Martinez, D. E. (2000). Unified nomenclature for the winged helix/forkhead transcription factors. Genes and Development, 14, 142-146.

[26] Robledo, S., Idol, R. A., Crimmins, D. L., Ladenson, J. H., Mason, P. J., \& Bessler, M. (2008). The role of human ribosomal proteins in the maturation of rRNA and ribosome production. RNA, 14, 1918-1929.

[27] Birney, E., Kumar, S., \& Krainer, A. R. (1993). Analysis of the RNA-recognition motif and RS and RGG domains: Conservation in metazoan pre-mRNA splicing factors. Nucleic Acids Research, 21, 5803-5816.

[28] Preiss, T. W., \& Hentze, M. (2003). Starting the protein synthesis machine: Eukaryotic translation initiation. Bioessays, 25, 1201-1211.

[29] Braddock, D. T., Louis, J. M., Baber, J. L., Levens, D., \& Clore, G. M. (2002). Structure and dynamics of KH domains from FBP bound to single-stranded DNA. Nature, 415, 1051-1056.

[30] Ruggero, D., \& Pandolfi, P. P. (2003). Does the ribosome translate cancer? Nature Review Cancer, 3, 
179-192.

[31] Sim, E. U. H., Bong, I. P. N., Balraj, P., Tan, S. K., Jamal, R., Sagap, I., et al. (2006). A preliminary study of differentially expressed genes in Malaysian colorectal carcinoma cases. Journal of Bioscience, 17, 19-37.

[32] Sikora, K., Chan, S., Evan, G., Gabra, H., Markham, N., Stewart, J., et al. (1987). C-myc oncogene expression in colorectal cancer. Cancer, 59, 1289-1295.

[33] Daftuar, L., Zhu, Y., Jacq, X., \& Prives, C. (2013). Ribosomal proteins RPL37, RPS15 and RPS20 regulate the Mdm2-p53-MdmX network. PLoS One, 8, 68667.

[34] Konstas, A. A., Shearwin-Whyatt, L. M., Fotia, A. B., Degger, B., Riccardi, D., Cook, D. I., et al. (2002). Regulation of the epithelial sodium channel by N4WBP5A, a novel Nedd4/Nedd4-2-interacting protein. Journal of Biological Chemistry, 277, 29406-29416.

[35] Cristillo, A. D., Nie, L., Macri, M. J., \& Bierer, B. E. (2003). Cloning and characterization of N4WBP5A, an inducible, cyclosporine-sensitive, Nedd4-binding protein in human T lymphocytes. Journal of Biological Chemistry, 278, 34587-34597.

[36] Ben-Neriah, Y. (2002). Regulatory functions of ubiquitination in the immune system. Nature Immunology, 3, 20-26.

[37] Grishin, N. V. (1998). The R3H motif: A domain that binds single-stranded nucleic acids. Trends in Biochemical Science, 23, 329-330.

[38] Fukita, Y., Mizuta, T. R., Shirozu, M., Ozawa, K., Shimizu, A., \& Honjo, T. (1993). The human Smubp-2, a DNA-binding protein specific to the single-stranded guanine-rich sequence related to the immunoglobulin mu chain switch region. Journal of Biological Chemisrty, 268, 17463-17470.

[39] Chen, N. N., Kerr, D., Chang, C. F., Honjo, T., \& Khalili, K. (1997). Evidence for regulation of transcription and replication of the human neurotropic virus JCV genome by the human S(mu)bp-2 protein in glial cells. Gene, 185, 55-62.

[40] Shieh, S. Y., Stellrecht, C. M., \& Tsai, M. J. (1995). Molecular characterization of the rat insulin enhancer-binding complex $3 \mathrm{~b} 2$. Cloning of a binding factor with putative helicase motifs. Journal of Biological Chemistry, 270, 21503-12508.

[41] Sebastiani, G., Durocher, D., Gros, P., Nemer, M., \& Malo, D. (1995). Localization of the Catf1 transcription factor gene to mouse chromosome 19. Mammalian Genome, 6, 147-148.

[42] Zhang, Q., Wang, Y. C., \& Montalvo, E. A. (1999). Smubp-2 represses the Epstein-Barr virus lytic switch promoter. Virology, 255, 160-170.

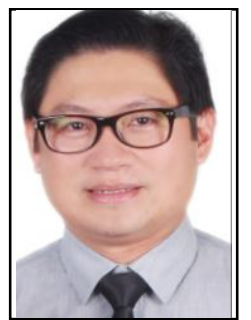

Edmund U. H. Sim was born in Malaysia on the 23 ${ }^{\text {rd }}$ December 1969, and obtained his Ph.D. in biochemistry from the University of Queensland, Brisbane, Australia in 2000. Prior to that, he obtained his B.Sc. Hons in genetics from the University of Malaya, Kuala Lumpur, Malaysia in 1993.

He is currently an associate professor at the Department of Molecular Biology, Universiti Malaysia Sarawak, and has been an academic at this university since 1994. He has 30 journal publications, to date. The latest publications include articles in Human Gene Therapy Methods (2014, Vol. 25 (1), 40-47), and Analytical Biochemistry (2013, Vol. 439 (2), 142-144). His research specialization is in cancer genetics.

He is an executive committee member of the Nasopharyngeal Carcinoma (NPC) Society of Malaysia, and a member of the Malaysian NPC Study Group. He is also a member of the Scientific and Technical Committee, 
and editorial review board (for Biological and Life Sciences) of the World Academy of Science, Engineering and Technology (WASET) since 2009. He is a member of the Advisory Committee for the School of Engineering, Computing and Science of the Swinburne University of Technology (Malaysia Campus). To date, he has acted as a manuscript reviewer for 9 international academic journals.

Chin-Ming Er is currently a Ph. D student at the Faculty of Civil and Environmental Engineering, Universiti Tun Hussein Onn Malaysia. He obtained his BSc. Hons in resource biotechnology from Universiti Malaysia Sarawak, Malaysia in 2009. 